\title{
A Search for Variable B Stars in the Northern Open Clusters
}

\author{
Andrzej Pigulski, Mikołaj Jerzykiewicz and Grzegorz Kopacki \\ Wroctaw University Observatory, ul. Kopernika 11, 51-622 Wrockaw, \\ Poland, Electronic mail: pigulski@ii.uni.wroc.pl
}

\begin{abstract}
In April 1994 we started a program of searching for variable B-type stars in young open clusters. The equipment consisted of a $60-\mathrm{cm}$ reflecting telescope and a CCD camera with a Johnson's B filter. Up to date we obtained nearly 1500 CCD frames of the central regions of four clusters: IC 4996, NGC 7235, NGC 7510, and NGC 654. In the range from $\mathrm{B} 0$ to $\mathrm{B} 5$, the photometric accuracy we achieve allows detection of short-period variability with amplitudes of about $5 \mathrm{mmag}$ or greater. In this note we present the short-period variable stars we found in NGC 7235 and NGC 7510 .
\end{abstract}

\section{Introduction}

With the discovery of the opacity-driven pulsational instability in models of Btype stars (see, e.g., Moskalik, these proceedings), the importance of finding $\beta$ Cephei variables and SPB stars in open clusters has shifted from mapping the instability domain in the HR diagram to (1) providing information about the clusters themselves and (2) identifying objects for detailed asteroseismological studies. The first possibility may lead to constraining the age and metal content of the clusters, the second will ultimately yield data for comparison with the theory of the interiors of hot stars.

\section{Equipment and reductions}

All observations were obtained in the Bialków station of the Wroclaw University Observatory. We used the Photometrics Star I camera with the Thomson TH $7883384 \times 576$ CCD chip, mounted in the Cassegrain focus of a $60-\mathrm{cm}$ reflecting telescope. The field was $4^{\prime} \times 6^{\prime}$. Most exposures were taken through a Johnson's $B$ filter. The exposure times ranged from 40 to 90 seconds.

The initial processing of the frames included corrections for bias, dark- and flat-fields. Stellar magnitudes were then derived by means of Stetson's (1987) Daophot II profile-fitting software. In the next step, differential magnitudes were calculated between pairs of stars. Variable stars were picked out at this stage. In the last step, we derived differential magnitudes once more, now using three nearby comparison stars for each variable we found. 


\section{NGC 7235}

There are 376 frames, obtained on 13 nights in the interval from JD 2449516 to JD 2449545. Out of the 80 brightest stars investigated so far, two show periodic variations. The numbers we assigned to them are 198 and 167 .

\#198. This is \#8 in the photoelectric sequence of Hoag et al. (1961). Our $B$ observations can be represented as a sum of two sinusoids with periods equal to $P_{1}=0 d 20305$ and $P_{2}=0 d 17800$. The amplitudes (half-ranges) amount to $12.6 \pm 0.6 \mathrm{mmag}$ and $10.1 \pm 0.6 \mathrm{mmag}$, respectively. We conclude that the star is a $\beta$ Cephei variable.

\#167 (47" north and $18^{\prime \prime}$ west of the second star in the photographic sequence of Hoag et al. 1961) exhibits a variation typical of the W Ursae Majoris eclipsing systems. The period is $0 d 2800$, implying component masses close to one solar mass. On the other hand, the star's $B$ magnitude is equal to 16.2 . If the star were a member of NGC 7235, this would translate into $M_{\mathrm{V}}=0.2$, corresponding to spectral type of B 9 . We conclude that \#167 is a foreground object.

\section{NGC 7510}

This cluster has been the subject of a recent CCD study by Sagar \& Griffiths (1991, henceforth SG). Our observations consist of 284 frames, taken on 11 nights in the interval from JD 2449547 to JD 2449569. About 100 brightest stars were checked for variability. Two turned out to be short-period variables.

SG 516 has a sinusoidal light-curve with a period of 0.1022 and an amplitude equal to $11.2 \pm 1.2 \mathrm{mmag}$.

According to Hoag et al. (1961), the photographic $U-B$ and $B-V$ of the star are equal to 0.58 and 0.81 , respectively. Using these data and the cluster's mean $E_{\mathrm{U}-\mathrm{B}}$ and $E_{\mathrm{B}-\mathrm{V}}$, we get $(U-B)_{0}=-0.19$ and $(B-V)_{0}=-0.25$. These numbers are clearly inconsistent. Note, however, that the ratio of the observed colour indices is very nearly equal to the slope of the reddening line in the twocolour diagram. Thus, a consistent pair of de-reddened colour indices would be obtained if SG 516 were assumed to be an early A-type star. We conclude that SG 516 is a foreground $\delta$ Scuti variable.

SG 101. Our observations can be represented by a period of 0.659 , or its $1 \mathrm{c} / \mathrm{d}$ alias, equal to $0 \mathrm{~d} 3976$. The data are insufficient to exclude either value.

Acknowledgments. This research was supported by KBN grant Nr 2 P304 00104 . M.J. gratefully acknowledges a travel grant from the Government of the Republic of South Africa.

\section{References}

Hoag, A. A., Johnson, H. L., Iriarte, B., Mitchell, R. I., Hallam, K. L., \& Sharpless, S. 1961, Publ. U.S. nav. Obs., 2nd ser., Vol. 17, part 7

Sagar, R., \& Griffiths, W. K. 1991, MNRAS, 250, 683

Stetson, P. B. 1987, PASP, 99, 191 